\title{
Patterns of invasive species spread in a landscape with a complex geometry
}

\author{
Weam Alharbi \& Sergei Petrovskii ${ }^{1}$ \\ Department of Mathematics, University of Leicester, \\ University Road, Leicester LE1 7RH, U.K.
}

\begin{abstract}
Patterns and rates of invasive species spread have been a focus of attention for several decades. Majority of studies focused on the species proliferation in a relatively uniform "open space" thus leaving aside the effects of the landscape geometry as given by size and shape of inaccessible areas. In this paper, we address this issue by considering the spatiotemporal dynamics of an alien species in a domain where two large uniform habitats are connected by a narrow corridor. We consider the case where the species is originally introduced into one of the habitats but not to the other. The alien species is assumed to be affected by a predator, so that mathematically our system consists of two coupled diffusion-reaction equations. We show that the corridor tends to slow down the spread: it takes the alien population an extra time to penetrate through the corridor, and this delay time can be significant in the case of patchy spread. We also show that a sufficiently narrow corridor blocks the spread; simple analytical estimates for the critical width of the corridor are obtained. Finally, we show that the corridor can become a refuge for the alien population. If considered on a longer timescale that includes species adaptation and/or climate change, the corridor may then become a source of a secondary invasion.
\end{abstract}

Keywords: corridor, invasion blocking, pattern formation, complex landscape, patchy invasion

\footnotetext{
${ }^{1}$ Corresponding author. Phone/fax: +44 116252 3916/3915, email: sp237@le.ac.uk
} 


\section{Introduction}

Biological invasion is a phenomenon that has a variety of important implications for ecology (in particular, through species extinctions and biodiversity loss), environment, human wellbeing, agriculture/aquaculture, and sometimes industries beyond agriculture $[15,39,44]$. For these reasons, it has been a focus of intense empirical and theoretical research for several decades $[8,41,57]$. Mathematical modelling is widely accepted as an efficient tool to study biological invasion [12, 21, 49]. Indeed, in the situation when replicated studies are hardly possible ${ }^{2}$, mathematical models along with computer simulations provide a virtual laboratory where the effect of various factors can be refined and analyzed and different hypotheses can be tested safely and at a relatively low cost.

In studies on biological invasion, a central question is how the alien species proliferates into space away from the place of its introduction. In its turn, it evokes the question as to what is the pattern of spread. For several decades, a paradigm of invasive species spread was a travelling population front. A large amount of theoretical work has been done in order to evaluate the speed of the front propagation $[2,10,18,51]$ and to reveal how the propagation can be affected by various factors $[4,20,30]$, in particular, by environmental heterogeneity $[3,14,32,48]$. The travelling front paradigm was eventually complemented by an alternative pattern of spread known as patchy invasion [11, 36, 38, 27] which is often observed in nature $[7,22,23]$ and predicted by various dispersal-growth models $[19,26$, $43,50]$. In the patchy invasion scenario, there is no population front and species spread into space occurs through the dynamics of separate disconnected population patches.

Whether it is a population travelling front or a patchy spread, previous studies on biological invasions have overwhelmingly focused on an idealized case of species spread in an unbounded space. (In case of a simulation study, the domain is bounded but can be chosen 'sufficiently large' in order to imitate an unbounded space.) Even that the effect of environmental heterogeneity was considered in a number of studies, e.g. see the references above, the role of inaccessible areas and hence the effect of landscape geometry on the rate of spread have remained poorly understood. Meanwhile, such a role is likely to be very important. One real world example is the invasion of grey squirrels in the UK. Whilst they successfully colonized most of England and Wales, the rate of their spread being in a good agreement with predictions of relevant mathematical models [4, 29], they have largely failed to spill over to central and northern Scotland, and the landscape geometry - as given by large areas that are not accessible to squirrels such as swamps or high mountain ranges - is thought to be a reason for that [56]. As another example, it was found in a study on the invasion of nutria in Iran that about one third of the country could potentially be colonized [9]. In reality, however, having been introduced about one hundred years ago to the sub-Caspian region, this species remains confined to largely the same area. A closer look reveals that different parts of the potential range are poorly connected (e.g. due to the effect of mountain ranges inaccessible to nutria), which is likely

\footnotetext{
${ }^{2}$ Not to mention the transient nature of the weather that makes it virtually impossible to reproduce the initial conditions, a repeated release of an alien species - potentially, a dangerous pest - would be highly controversial and possibly illegal.
} 
to limit species dispersal.

In this paper, we consider the effect of the landscape geometry on the invasive species spread by means of mathematical modelling and computer simulations. The "complexity" of the landscape geometry (as is relevant in the context of this study) is represented by a dumbbell-like, "H-shaped" spatial domain consisting of two large habitats connected by a narrow passage or corridor. The initial conditions correspond to the situation where the alien species is present in one of the habitats but not in the other. We are especially interested in the following questions:

- How much a narrow passage can slow down the spread? Can a sufficiently narrow passage block the spread completely so that the invading population would never spill over to the other side?

- Depending on species traits, invasive spread into open space is known to follow a few different scenarios (see Fig. 1 below). Can the effect of the passage change the invasion scenario, e.g. turning a propagating population front into patchy spread (or visa versa)?

- Can the effect of the passage depend on the invasion scenario (e.g. slowing down the spread in one case but not having much effect in another)?

It has been shown in our earlier work [1] that the success of the species invasion into the second habitat depends on the width and length of the corridor as well as demographic parameters such as the maximum growth rate and the strength of the Allee effect. However, the effect of the dynamical complexity on the invasive species spread, in particular due to interspecific interactions and pattern formation, has remained obscure. Here we show that the dynamical complexity can affect the invasion success significantly. In particular, we show that the effect of the corridor on the invasion success is significantly different depending on whether the invasive species spreads following the travelling population front scenario or the patchy invasion scenario. We also show that a corridor that blocks the species spread can become a refuge for the alien species and can subsequently become a source of a secondary invasion.

\section{Model}

The choice of the mathematical model is a somewhat subtle issue [25, 31, 34]. From a biological standpoint, it might be tempting to account in detail for a variety of biological interactions that the invasive species is experiencing during its spread, e.g. interactions with all species of the native community. A corresponding model is likely to be very complicated. Such a model would be difficult to parameterize as the information about the feedbacks and functional responses as well as parameter values is usually meagre and incomplete. Moreover, the higher the complexity of the model is, the more difficult it may be to reveal the general properties of the phenomenon as they may become obscure by specific details. Since in this paper our goal is to look into general properties of the 
dynamics rather than to study a particular case, the model should not be too complicated. However, it should not be too simple either as some important features of the invasion dynamics - such as, for instance, pattern formation [11, 24, 36, 46, 47, 50] - can be missing altogether due to the insufficient complexity of the model.

We therefore look for a model of an intermediate complexity. Arguably, an appropriate model is a prey-predator system. On the one hand, it obviously takes into account only some common, basic interactions, i.e. how the population dynamics of the alien species (regarded in this context as a prey) is affected by its predator where the predator can be a native species or can be introduced as a biological control agent, cf. [16, 33, 38]. On the other hand, a spatially explicit prey-predator model allowing for the population dispersal is known to have a complicated spatiotemporal dynamics [21, 24], its "realistic" properties being consistent with at least some field observations [11, 30].

A general prey-predator system is described by the following coupled diffusion-reaction equations $[28,30,46]$ :

$$
\begin{gathered}
\frac{\partial U(\mathbf{r}, T)}{\partial T}=D_{1} \nabla^{2} U(\mathbf{r}, T)+F(U)-f(U, V), \\
\frac{\partial V(\mathbf{r}, T)}{\partial T}=D_{2} \nabla^{2} V(\mathbf{r}, T)+\kappa f(U, V)-M V,
\end{gathered}
$$

where $U(\mathbf{r}, T)$ and $V(\mathbf{r}, T)$ are, respectively, the prey and predator population densities at time $T$ and position $\mathbf{r}=(X, Y), D_{1}$ and $D_{2}$ are the corresponding diffusion coefficients, $\kappa$ is the food utilization coefficient. Function $F(U)$ is the growth rate of the prey, $f(U, V)$ describes predation, and $M$ is the predator mortality rate. A particular choice of functions $F(U)$ and $f(U, V)$ may vary depending on the species traits. We assume that the predator trophic response is of the Holling type II and choose the parametrization accordingly [28]:

$$
f(U, V)=\frac{A U V}{U+H},
$$

where $A$ quantifies the predation rate and $H$ is the half-saturation prey density.

With regard to the prey growth, there is considerable evidence that the Allee effect is common in the population dynamics [5, 6]. Correspondingly, we assume that the alien species is affected by the strong Allee effect and consider the following parametrization for its growth rate [20]:

$$
F(U)=G U\left(U-U_{A}\right)(K-U),
$$

where $G$ is a coefficient related to the maximum per capita growth rate $g$ as $G=4 \mathrm{~g} /(K-$ $\left.\left.U_{A}\right)\right)^{2}[20], K$ is the carrying capacity, and the Allee threshold density $U_{A}\left(0<U_{A}<K\right)$ is a parameter quantifying the strength of the Allee effect [30, 55].

Equations (1-2) with (3-4) contain a large number of parameters, which makes their numerical investigation cumbersome. However, if we choose new, dimensionless variables as $u=U / K, v=V /(\kappa K), t=a T$ where $a=A \kappa K / H, x=X\left(a / D_{1}\right)^{1 / 2}$ and $y=$ $Y\left(a / D_{1}\right)^{1 / 2}$, Eqs. (1-2) turn to the following:

$$
\frac{\partial u(x, y, t)}{\partial t}=\left(\frac{\partial^{2} u}{\partial x^{2}}+\frac{\partial^{2} u}{\partial y^{2}}\right)+\gamma u(u-\beta)(1-u)-\frac{u v}{1+\alpha u},
$$




$$
\frac{\partial v(x, y, t)}{\partial t}=\epsilon\left(\frac{\partial^{2} u}{\partial x^{2}}+\frac{\partial^{2} u}{\partial y^{2}}\right)+\frac{u v}{1+\alpha u}-\delta v
$$

where $\alpha=K / H, \beta=U_{A}, \gamma=G H K /(A \kappa), \delta=M / a$ and $\epsilon=D_{2} / D_{1}$ are new (dimensionless) parameres. Properties of dimensionless solutions $u$ and $v$ therefore depend on five dimensionless combinations rather than on each of the nine original parameters separately.

Biological invasion usually starts with an introduction of a (small) number of individuals of an alien species into a certain location inside a new ecosystem [49]. Immediately after the introduction, the population density of the alien species is only non-zero inside a certain (small) area. Therefore, in order to account for the biological invasion, Eqs. (5-6) must be supplemented with initial conditions described by functions of a finite support. Here we consider the initial conditions as follows:

$$
\begin{aligned}
& u(x, y, 0)=u_{0} \text { if } x_{11}<x<x_{12} \text { and } y_{11}<y<y_{12} \\
& \text { otherwise } u(x, y, 0)=0, \\
& v(x, y, 0)=v_{0} \text { if } x_{21}<x<x_{22} \text { and } y_{21}<y<y_{22} \\
& \text { otherwise } v(x, y, 0)=0,
\end{aligned}
$$

where $u_{0}, v_{0}$ the initial prey and predator densities, and parameters $x_{i j}$ and $y_{i j}(i, j=1,2)$ determine the initially occupied area.

\section{Simulations and results}

Equations (5-6) with the initial conditions (7-8) were solved numerically by finite-differences. In most cases we used the simple explicit scheme. The mesh steps were chosen as $\Delta x=\Delta y=0.4$ and $\Delta t=0.03$; it is readily seen that they satisfy the CourantFriedrichsLewy stability condition [42]. Having performed simulations for a few other values of the mesh steps, we checked that the above values are sufficiently small to avoid numerical artifacts.

Although our main goal in this paper is to study the effects of a complex geometry, we begin with a simple case when Eqs. (5-6) are considered in a square domain. The domain has size $L$ so that $0<x<L$ and $0<y<L$. At the boundary of the domain the Neumann-type no-flux conditions are imposed. For any given parameter set, the simulation is stopped either just before or immediately after the spreading population reaches the domain boundary. In this way, the effect of the boundary conditions on the population dynamics inside the domain is minimized; correspondingly, the population spread imitates the spread in an open (unbounded) space.

Depending on the parameter values such as the predator mortality rate $\delta$ and/or the Allee threshold $\beta$, the prey-predator system (5-6) is known to exhibit different patterns of the population spread $[24,27,54]$. Having chosen $\delta$ as the controlling parameter, a brief visual overview of the patterns is shown in Fig. 1 where the results were obtained in a square domain $200 \times 200$ for parameters $\epsilon=1, \gamma=3.9, \alpha=0.1, \beta=0.2$, the initial 
conditions (7-8) with $u_{0}=1, v_{0}=0.2, x_{11}=85, x_{12}=105, x_{21}=85, x_{22}=95, y_{11}=100$, $y_{12}=105, y_{21}=95$ and $y_{22}=115$, and different values of $\delta$. Here and below, only the distribution of prey is shown as the distribution of predator exhibits similar features. Unless the initially inhabited domain is very small so that the alien population goes extinct

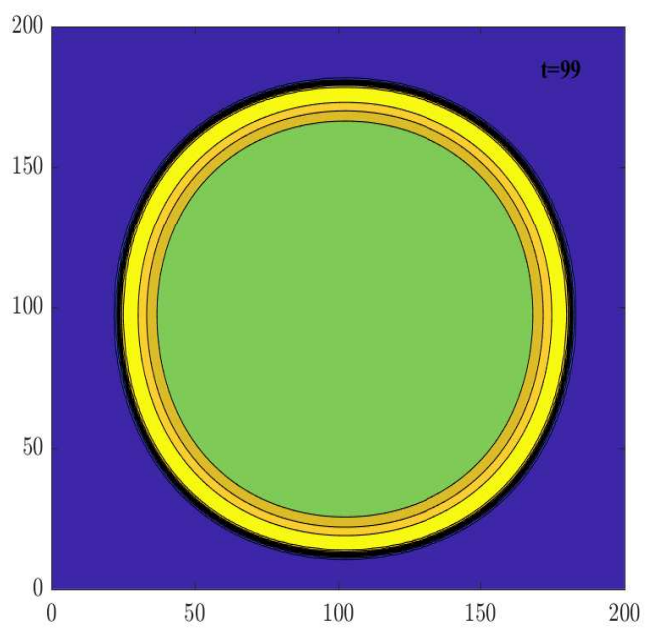

(a)

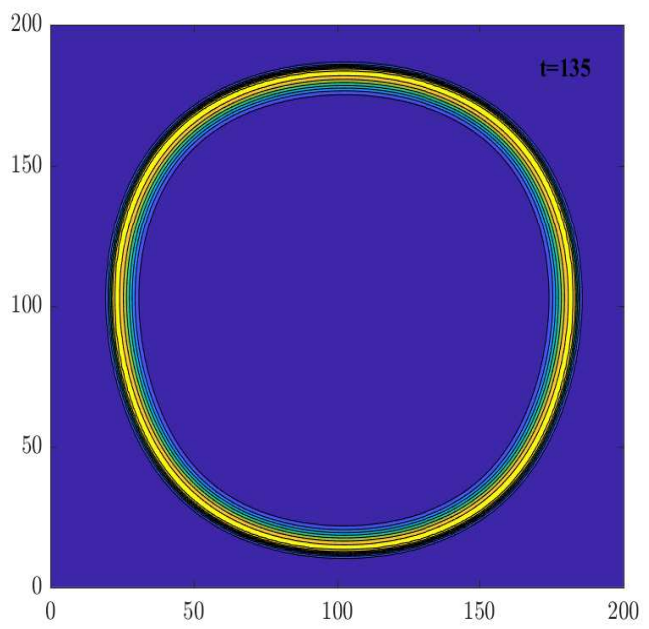

(c)

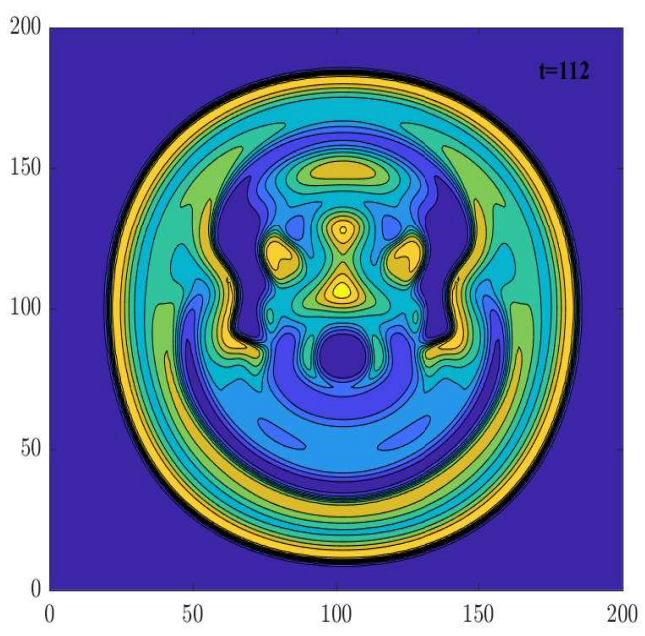

(b)

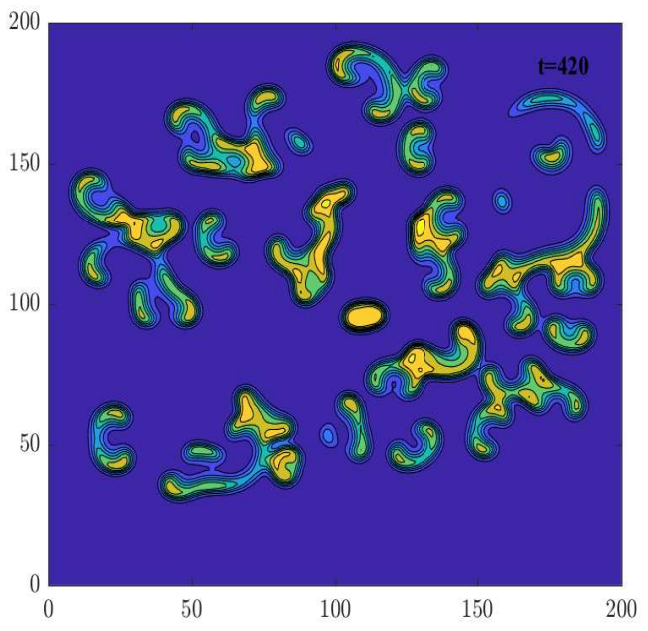

(d)

Figure 1: Snapshots of the spatial distribution of prey (i.e. the alien species) illustrating different scenarios of invasive spread as predicted by Eqs. (5-6) obtained in a square domain $300 \times 300$ for parameters (a) $\delta=0.63$, (b) $\delta=0.51$, (c) $\delta=0.43$ and (d) $\delta=0.37$. Other parameters are given in the text. Yellow color for high population density, green and light blue for intermediate values, dark blue for zero density. 


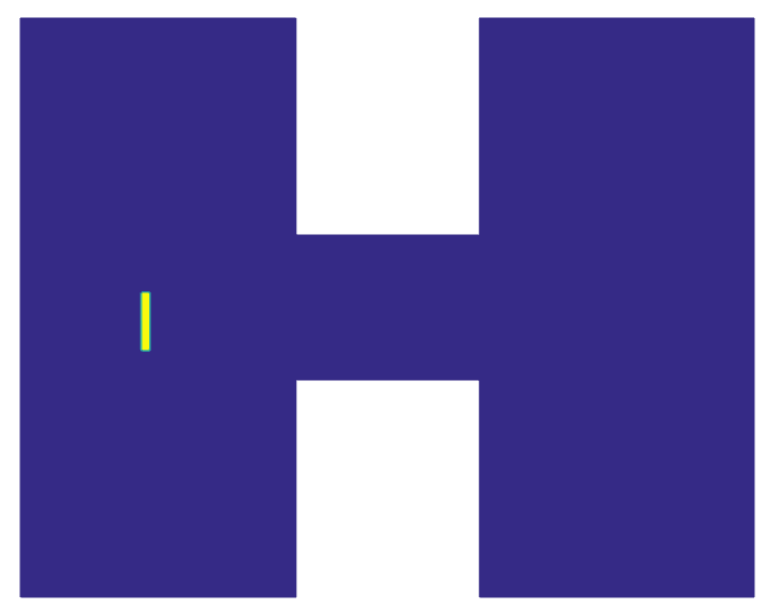

Figure 2: A sketch of the H-shaped domain consisting of Habitat 1 at the left and Habitat 2 at the right connected by a narrow passage (corridor). The alien species is introduced in Habitat 1, the initially inhabited area is shown by a small yellow rectangle.

due to the strong Allee effect [20], a somewhat trivial case that we do not consider here, the alien population spreads into the empty space away from the place of its introduction. This spread can follow the population front propagation scenario (Fig. 1a,b,c) with or without pattern formation in the wake or the patchy invasion scenario [36, 38] (Fig. 1d) where the population spread is not preceded by a travelling front propagation.

A practically important question is how high is the rate of invasive spread. It can be rephrased as follows: for a given location of the alien species introduction, how fast the invading species will arrive at a given location elsewhere? In a spatially uniform system, this question is linked to the question about the speed of the propagation of the travelling population front ${ }^{3}$ : indeed, once the speed is known, a common sense estimate is time = distance/speed. However, it is intuitively clear that this simple argument is unlikely to remain valid in a case where the invasive spread takes place in a landscape of a complicated geometry where the spread can possibly be slowed down or block altogether by, for instance, the existence of narrow valleys or 'corridors'.

In order to make an insight into possible effects of the corridors, we now consider a domain of a more complex shape, namely, H-shaped where two habitats (subdomains) of a rectangular shape are connected by a narrow passage or corridor. For the initial conditions, we consider the situation where the alien species is introduced to a certain location close to the center of the left-hand side habitat (which we for convenience also call Habitat 1); see Fig. 2. For the boundary conditions, we consider the Neumann-type zero-flux condition along the whole boundary. One can expect that during the first stage

\footnotetext{
${ }^{3}$ The situation is somewhat more complicated in the case of patchy invasion or in the case where the invasive species employs a long-distance dispersal. In the former case there is no front and hence the definition of the speed is somewhat ambiguous [27], in the latter case the front can have variable speed $[17]$.
} 
of invasion, i.e. before the invading population reaches the boundary of Habitat 1, the dynamics should be essentially the same as in a domain of a simple shape or in an infinite space. Once the invading population reaches the entrance to the corridor, however, it becomes a new, different problem and the question arises as to whether the population spills over to the right-hand side habitat (which we call Habitat 2) for given length and width of the corridor and for a given pattern of spread, cf. Fig. 1.

The case of the population spread with a uniform spatial population distribution behind the invasive front (Fig. 1a) is similar to the invasive spread in a single species model considered in [1]; we do not reconsider it here. We therefore begin with a more interesting case where invasion into a homogeneous space takes place via the propagation of the population front with spatiotemporal pattern formation in the wake (cf. Fig. 1b). The results shown in Fig. 3 are obtained for the parameters of the corridor as $(\lambda, w)=(10,2.8)$ where $\lambda$ is the length and $w$ is the width. In the first stage of invasion, the population front propagates fast, apparently with the same speed as in an infinite space [21]. Once it reaches the entrance to the corridor, the spread slows down considerably: it takes the population about 100 extra time units to penetrate through the corridor, cf. the top-right and middle-left panels in Fig. 3. Eventually, the population spills over to Habitat 2 and invade over the rest of the domain, see the bottom row in Fig. 3.

Interestingly, the corridor affects the pattern of spread. The spread of the invading population in Habitat 2 is accompanied by the formation of irregular asymmetric patterns in the wake of the propagating front, which apparently is a result of the asymmetry in the initial conditions. However, the population distribution behind the front in Habitat 2 exhibits a perfect circular symmetry, e.g. see the bottom-left panel in Fig. 3.

However, spilling over after a delay caused by the corridor is not the only possibility. The system can also exhibit a qualitatively different dynamics resulting in an invasion failure. Simulation results (not shown here for the sake of brevity) obtained for different parameters of the corridor reveal that the dynamics depend strongly on the size of the corridor, in particular on its width. An increase of the corridor width will not change the dynamics much resulting only in a decrease of the time required for the population to penetrate through. However, a decrease of the corridor width will eventually result in invasion blocking: as soon as the width is smaller than a certain critical value, the invading population is blocked in the passage and never spills over to Habitat 2.

In order to check whether the above situation is general or pattern-specific, we then performed simulations for a value of $\delta$ where the species spread in an open space takes place via the propagation of a solitary ring (see Fig. 1c). The results are shown in Fig. 4. It is readily seen that the population dynamics follows a similar scenario. In the first stage, the population spreads away from the place of its original introduction in the same manner as in an open space. Once the front reaches the entrance to the passage, the spread slows down; it takes it about 40 time units to penetrate through to enter Habitat 2. (Interestingly, this time appears to about twice shorter than in the previous case.) Once it spills over, the population spreads over Habitat 2 in a similar way as before. One difference from the corresponding pattern of spread in an open space is that a fraction of the population settles down inside the passage (this is well seen in the middle row 
of Fig. 4) and works as a source to generate a sequence of population fronts (spreading rings) into both habitats. As well as above, a successful spill-over through the corridor only happens if its width is not too narrow. Once the width becomes less than a certain critical value, the population is blocked in the passage and never penetrates to the other
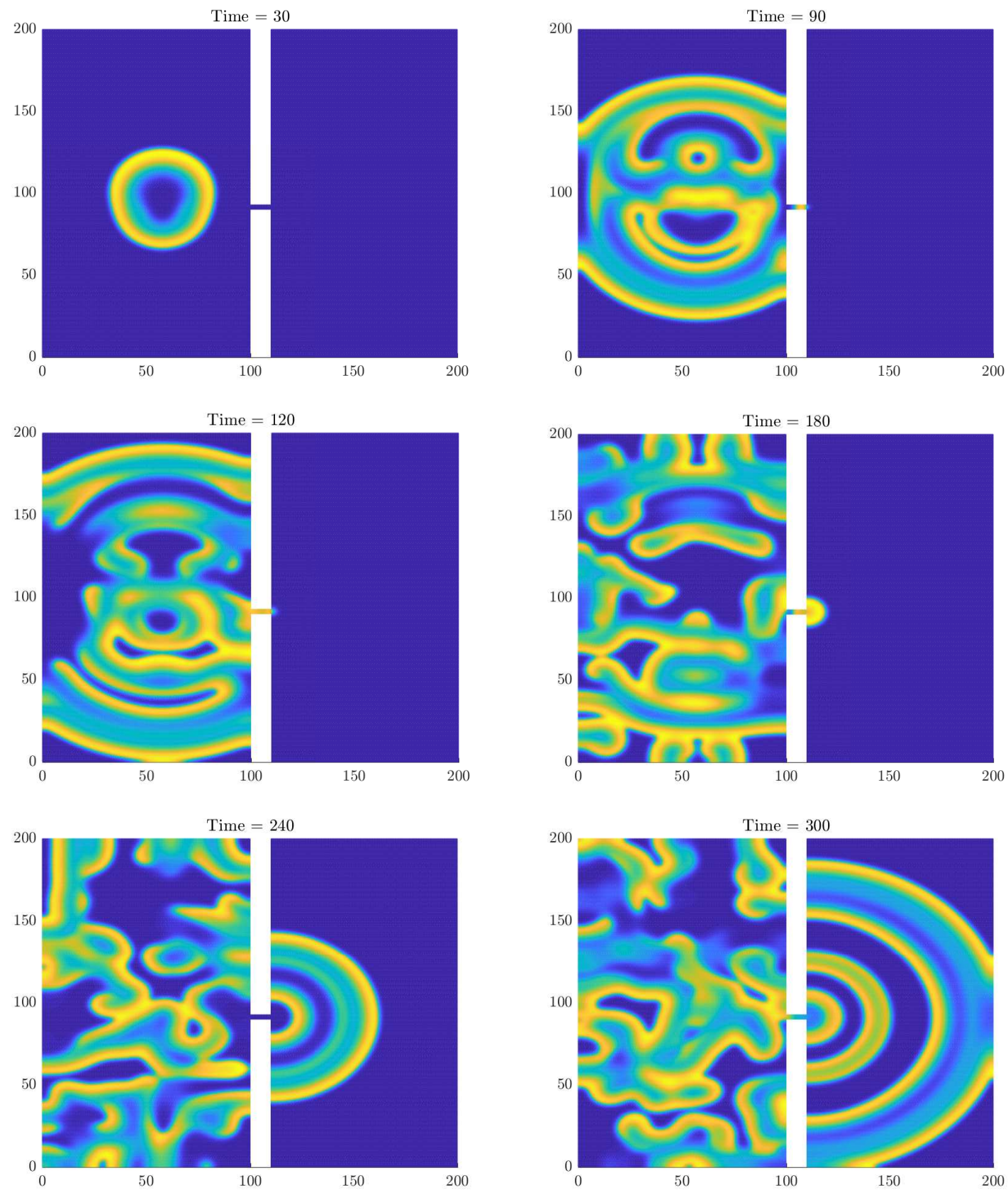

Figure 3: Snapshots of the spatial distribution of prey at different moments calculated for parameters $\epsilon=1, \gamma=3.9, \alpha=0.1, \beta=0.2$ and $\delta=0.5$ and initial condition (7-8) with $u_{0}=1, v_{0}=0.2, x_{11}=85.2, x_{12}=105.2, x_{21}=85.2, x_{22}=95.2, y_{11}=55.2$, $y_{12}=60, y_{21}=50, y_{22}=70$ (note that $x$ is the vertical axis and $y$ is the horizontal one). Parameters of the corridor are $(\lambda, w)=(10,2.8)$. Yellow for high population density, dark blue for the zero density, green for intermediate density. 
side.

Finally, we performed simulations for the value of $\delta$ where the alien population spreads into space following the patchy invasion scenario (see Fig. 1d). The results are shown in Fig. 5. The dynamics exhibit features similar to those observed in Figs. 3 and 4. The population first spreads from the place of its original location in exactly the same manner as it would happen in a domain of a simpler shape or in an open space. Once it approaches the entrance to the passage, it is delayed for a considerable time before it spills over to
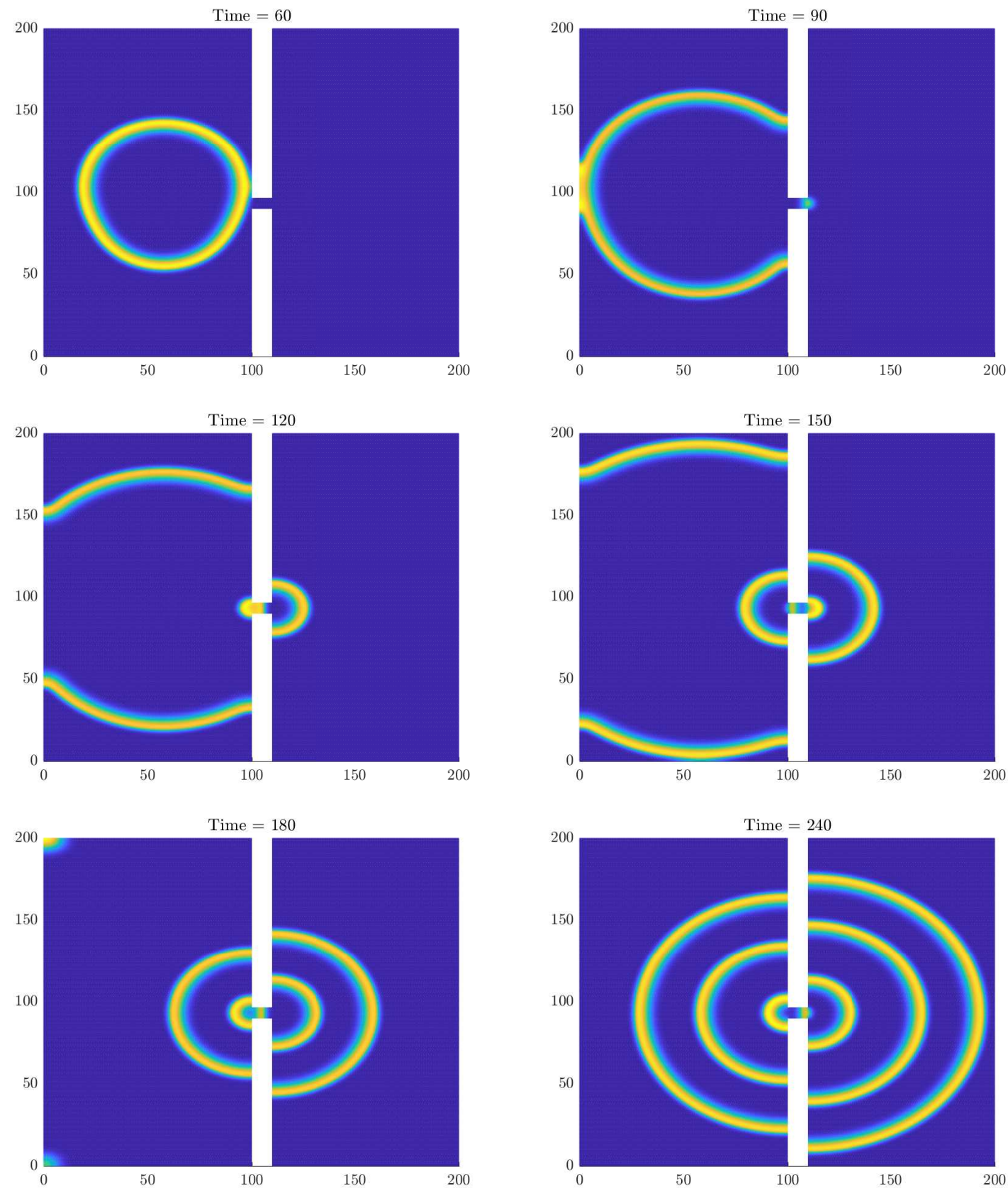

Figure 4: Snapshots of the spatial distribution of prey at different moments calculated for parameters $\delta=0.42$ and $($ lenght,$w)=(10,6.4)$, other parameters and color coding are the same as in Fig. 3. 
Habitat 2. Note that the this delay is two orders of magnitude longer than it was in the previous cases. An explanation of this lies in the absence of the population front: because of the chaotic movement of the patches in space [27], it takes an extra time for a patch to hit the entrance to the passage compared to the directional propagation of the front. Eventually, if the corridor's width is greater than a certain critical value, the population spreads through the passage and invades over Habitat 2 (as in the bottom row of Fig. 5).
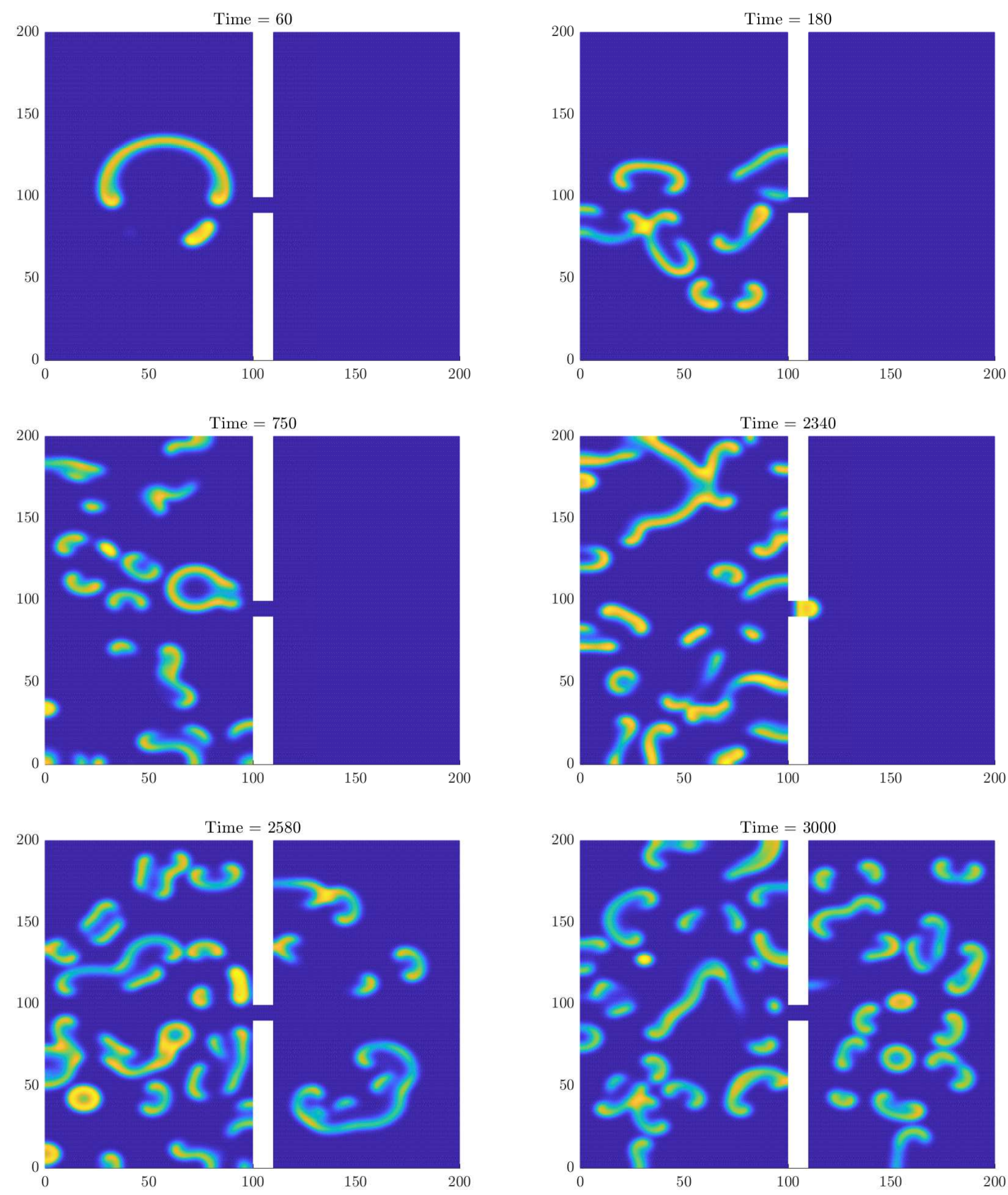

Figure 5: Snapshots of the spatial distribution of prey at different moments calculated for parameters $\delta=0.37$ and (lenght, $w)=(10,9.2)$, other parameters and color coding are the same as in Fig. 3. Note the very long time that it takes the invading population to spill over to the right-hand side habitat. 
However, in case the corridor is not wide enough the population is blocked and never penetrates to the other side (not shown here).

\subsection{Propagation blocking}

In order to make a more detailed insight into the existence of the corridor's critical width and how its value depends on the predator mortality $\delta$ (considered as a controlling parameter) and hence on the type of species spread, we performed extensive computer simulations with different values of $w$ and $\delta$ keeping other parameters the same as above. For each parameter set, the code was run up to $t=4000$. The results are shown in Fig. 6 . We therefore observe that the critical width exhibits a non-monotone dependence on the predator mortality but tends to increase with a decrease in $\delta$.

An analytical estimation of the critical width appears to be possible. Using the comparison principle for nonlinear partial differential equations [40, 53], it is readily seen that the fllowing single-species model:

$$
\frac{\partial u(x, y, t)}{\partial t}=\left(\frac{\partial^{2} u}{\partial x^{2}}+\frac{\partial^{2} u}{\partial y^{2}}\right)+\gamma u(u-\beta)(1-u)
$$

i.e. Eq. (5) without the last term in the right-hand side, is the upper bound for the preypredator system (5-6). However, for the single-species model a simple analytical estimate of the critical width can be easily obtained. Indeed, once the front arrives to the end of the passage and starts spilling into Habitat 2, it is of approximately circular shape

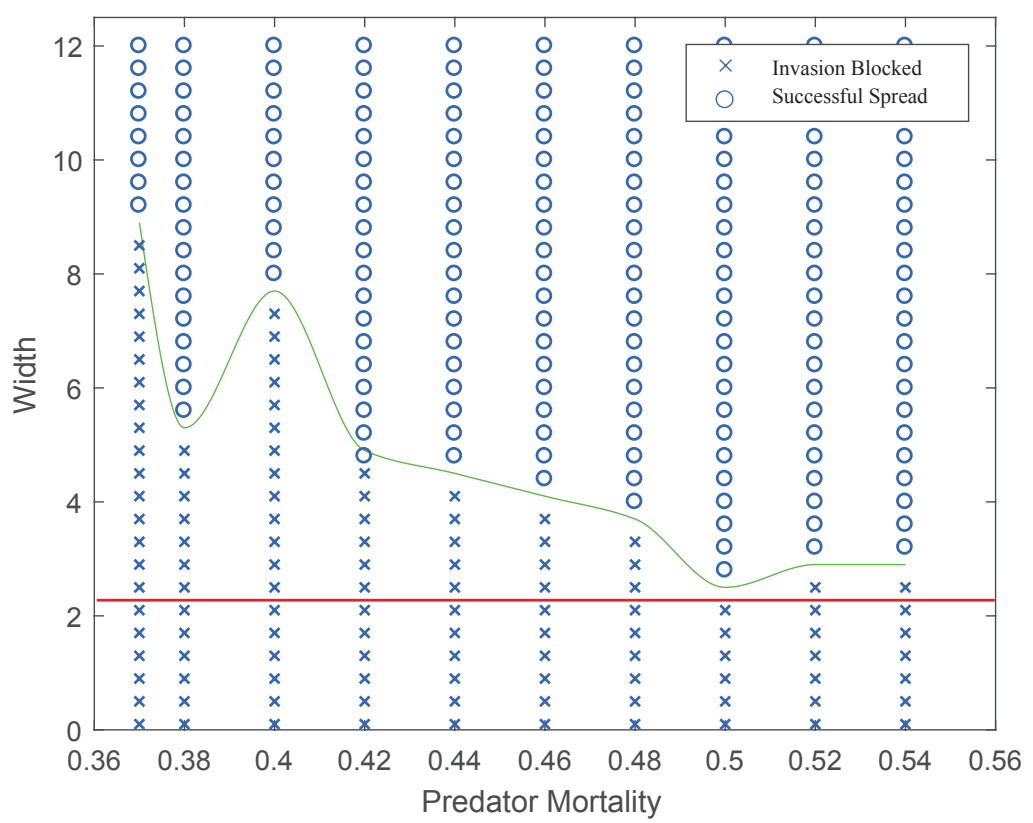

Figure 6: Parameter plane $(w, \delta)$, other parameters are the same as above. For the parameters from above the green curve (obtained in simulations) the population spills over and invade Habitat 2, for the parameters from below the curve the population is blocked in the passage. Red line shows the analytical estimate (14). 
(consistent with the numerical simulations above, e.g. see the middle-left panel in Fig. 3 and the middle-right panel in Fig. 5). For a circular propagating front of the radius $R$ in a $2 \mathrm{D}$ system, its speed $c(R)$ is known to be related to the speed $c$ of the corresponding $1 \mathrm{D}$ front as

$$
c(R)=c-\frac{D}{R}
$$

e.g. see [54]. For the front confined at the exit of the passage, $R=w / 2$. Since the front is blocked, $c(R)=0$. Equation (10) then turns into the following:

$$
c-\frac{2 D}{w}=0
$$

For a scalar diffusion-reaction equation with the population growth parameterized by a cubic polynomial, the analytical expression for the speed of the front propagation is well-known:

$$
c=\sqrt{\frac{D G}{2}}\left(K-2 U_{A}\right),
$$

e.g. see [21]. From (11) and (12), we therefore obtain:

$$
w_{c r}=\sqrt{\frac{8 D}{G}} \frac{1}{\left(K-2 U_{A}\right)},
$$

or, in dimensional variables,

$$
w_{c r}=\frac{2 \sqrt{2}}{(1-2 \beta) \sqrt{\gamma}} .
$$

For the parameters of Fig. 6 , the estimate $(14)$ gives $w_{c r} \approx 2.3$ which agrees well with the simulation results.

Now, a question can arise here as to how sensitive the above results are to the boundary conditions along the corridor. Indeed, it is well known that the critical behaviour of

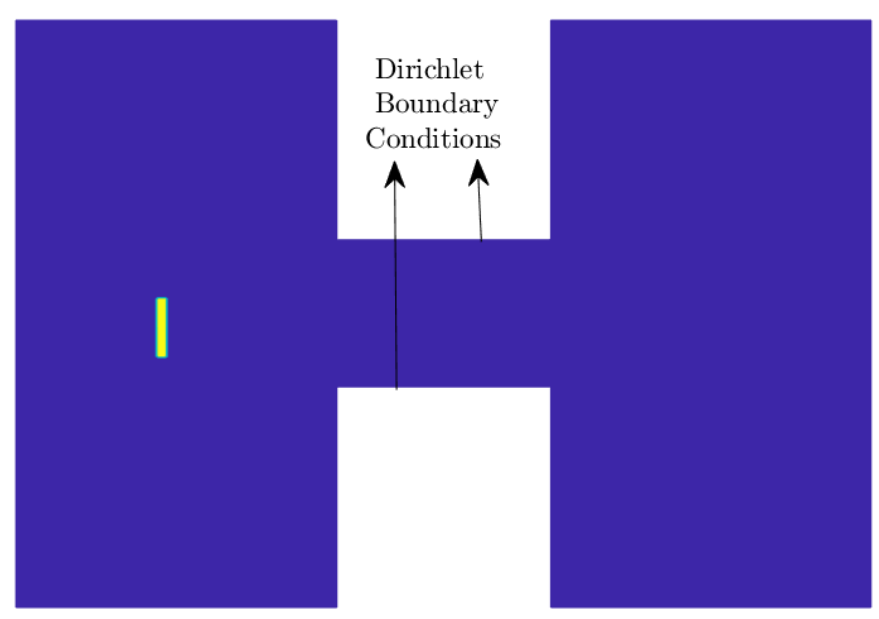

Figure 7: Sketch of an H-shaped habitat with Dirichlet boundary conditions at the sides of the corridor. 
diffusion-reaction systems is generally sensitive to the boundary conditions; for instance, the solution of the critical size problem is essentially different in case of the Neumann (zeroflux) and Dirichlet (zero-density) conditions. In order to make an insight into this issue, we now consider the same H-shaped domain as above but with the Dirichlet zero-density conditions along the passage boundary; see Fig. 7. We mention here that, compared to the zero-flux condition, the zero-density boundary condition corresponds to a somewhat different situation. Instead of a narrow canyon (connecting two large valleys) where the population density can build up in the vicinity of the canyon wall, one can think, for instance, of a narrow ridge (e.g. connecting two mountain plateaus) where a casual step over the cliff would result in death - hence, the zero population density.

Having performed extensive computer simulations in the domain with the zero-density condition in the passage (Fig. 7), we obtain that the dynamics of the system is qualitatively the same as in the previous case of the zero-flux conditions. In particular, we obtained that for all invasion regimes (as in Fig. 1) the invading population spills over to Habitat 2 if the corridor width is large enough and is blocked if the corridor is too narrow. Dependence of the critical width on the predator mortality is shown in Fig. 8. Therefore, as well as in the previous case we observe non-monotone dependence of $w_{c r}$ on $\delta$ with a tendency for the critical width to increase with a decrease in $\delta$.

As well as above, it appears possible to obtain a simple analytical estimate of the critical corridor width, although the argument and calculations are different. We recall that the single-species model is an upper bound for the prey-predator model, so a condition of

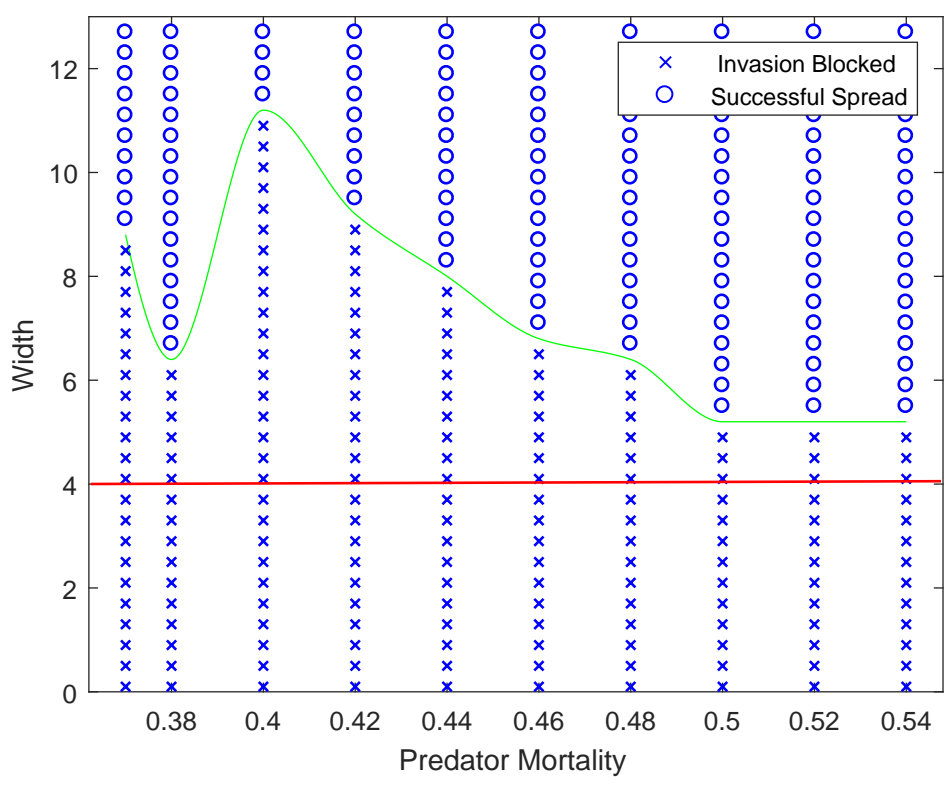

Figure 8: Parameter plane $(w, \delta)$ in case of the Dirichlet zero-density conditions at the boundary of the corridor. Other parameters and boundary conditions are the same as above. For the parameters from above the green curve (obtained in simulations) the population spills over and invade Habitat 2, for the parameters from below the curve the population is blocked in the passage. Red line shows the analytical estimate (17). 
population propagation blocking in the model (9) will be a sufficient condition of the propagation blocking in model (5-6). Consider the case of a long corridor. Consider the situation where the invasive species has penetrated through the corridor and successfully spilled over to Habitat 2. In this case, in the large time limit the population density profile across the corridor does not depend on its position along the corridor as far as the position is sufficiently far away from the corridor ends. The problem then becomes onedimensional as the population density depend only on the coordinate across the corridor but not along the corridor. However, if considered in the 1D settings, this turns into the classical critical size problem (e.g. see Section 3.1 in [21]), that is, how large the domain must be to ensure the survival of the population inside. For the growth rate described by a cubic polynomial, its analytical solution is unknown. However, if we consider an upper bound of the growth rate as

$$
\gamma u(u-\beta)(1-u) \leq g u \quad \text { for any } \quad u \geq 0
$$

where $g$ is the maximum per capita growth rate, see the lines below Eq. (4), the expression for the critical size in the corresponding linear problem is well known:

$$
w_{c r}=\pi \sqrt{\frac{D}{g}}
$$

and in the dimensionless variables:

$$
w_{c r}=\frac{2 \pi}{(1-\beta) \sqrt{\gamma}}
$$

For the parameters used in simulations, the estimate $(17)$ gives the value $w_{c r} \approx 4$, which appears to be in good agreement with numerical results; see Fig. 8.

Note that the mechanism of spread blocking is somewhat different in the two cases. For the zero-density conditions at the boundary of the passage, the population is blocked inside the passage (in a manner similar to how a subcritical size of the inhabited domain results in the population extinction). For the zero-flux conditions, however, the population cannot be blocked inside (except for the trivial case where $\beta>0.5$ ); instead, its propagation is blocked at the exit of the passage.

Finally, we want to emphasize here that both (14) and (17) are sufficient conditions of propagation blocking but not criteria. Once $w \leq w_{c r}$, the propagation will necessarily be blocked by the passage; however, $w>w_{c r}$ does not guarantee that the population will spread into Habitat 2. In terms of the diagrams shown in Figs. 6 and 8, it means that the whole of the red line lies in the parameter range where the population spread is blocked in the prey-predator system (i.e. in the area marked by crosses).

\subsection{Sensitivity of the patchy spread to the initial conditions}

From the four invasion scenarios shown in Fig. 1, perhaps the patchy invasion poses the biggest challenge. The absence of a continuous propagating population front leads to a certain ambiguity in the definition of the extent of the invaded area [27]. Similarly, it 


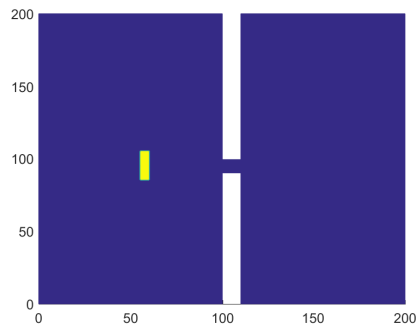

(a)

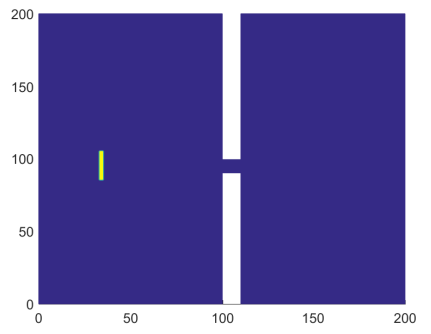

(b)

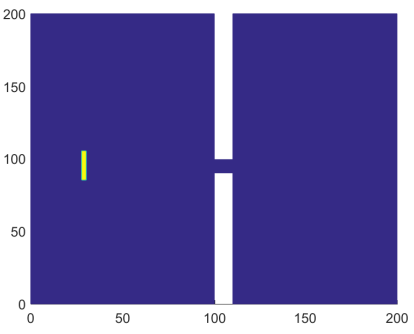

(c)

Figure 9: Snapshots of different initial predator population distributions used in simulations: (a) (IC-1) $y_{11}=55, y_{12}=60, y_{21}=50$ and $y_{22}=70$; (b) (IC-2) $y_{11}=32.8$, $y_{12}=35.2, y_{21}=30$ and $y_{22}=40 ;(\mathrm{c})(\mathrm{IC}-3) y_{11}=27.6, y_{12}=30, y_{21}=25.2$ and $y_{22}=35.2$. The initial conditions for the prey and other parameters are given in the text.

introduces an uncertainty as to when exactly, for a given set of the initial conditions, the population reaches the entrance to the passage. It is not enough for the expanding 'envelope' (e.g. the location of the foremost patch, cf. [27]) to reach the entrance to the corridor; in order for the population to start penetrating through the corridor, there must be one of the patches that actually hits the entrance. However, since the spatiotemporal population dynamics during the patchy invasion is known to be chaotic, the movement of the patches is effectively random. In its turn, the exact location of the patches depends on the initial population distribution. Therefore, one can expect that, altogether, the time that takes the population to arrive at the passage entrance and to penetrate to the other side can vary significantly.

In order to make a more quantitative insight into this issue, we therefore performed simulations for a few different initial conditions choosing the demographic parameters in the range where the patchy invasion occurs $[11,27,38]$. Specifically, we use $\gamma=3.9, \alpha=$ $0.1, \beta=0.2$ and $\delta=0.37$. The parameters of the corridor are chosen as $(\lambda, w)=(10,9.2)$. For the initial conditions, we fix the initial population densities as $u_{0}=1.2$ and $v_{0}=0.2$, and the initial distribution of prey as $x_{11}=85.2, x_{12}=105.2, x_{21}=85.2, x_{22}=95.2$, and consider a few different initial distribution of the predator; see Fig. 9.

For the above parameters and for each of the three initial distributions, the population spread from the place of its introduction generally follows the scenario shown in Fig. 5 (we do not show the results for the sake of brevity). We first consider the case with the zero-flux conditions on the passage boundary. In each simulation run, we pick the time required for the population to reach Habitat 2, e.g. the 'moment' when the population has penetrated through the passage and starts spilling over into the open space on the other side, e.g. as shown in the middle-right panel in Fig. 5. The results are presented in Table 1. It is readily seen that the time varies quite significantly; in fact, by an order of magnitude. We then repeat simulations for the same parameters and the same sets of initial conditions but imposing the zero-density conditions at the passage boundary. As 
well as in the previous case, the spill-over time shows a considerable variation between different initial conditions.

\subsection{Corridor as a refuge}

Finally, we report on a curious invasion scenario that occurs in a certain range of the parameters of the corridor. Figure 10 shows snapshots of the prey distribution obtained for the demographic parameters chosen as $\gamma=6.25, \alpha=0.1, \beta=0.2$ and $\delta=0.5$. The parameters of the corridor are $(\lambda, w)=(10,2.8)$ and the initial conditions are given by $(7-8)$ with $u_{0}=1.0, v_{0}=0.2, x_{11}=85.2, x_{12}=105.2, x_{21}=85.2, x_{22}=95.2, y_{11}=55.2$, $y_{12}=60, y_{21}=50$ and $y_{22}=70$. The boundary condition are zero-flux along the whole boundary of the domain (including the passage). Apparently, for these parameters the population spreads into an open space via ring-shaped travelling fronts; see the top-left panel in Fig. 10. After the population has propagated through the passage, a number of travelling concentric fronts is produced on both sides of the passage; see the middle row in Fig. 10. Eventually, the fronts go away (cf. the bottom row in Fig. 10) and the alien population eventually goes extinct in the most of the domain - except for the passage. A close inspection reveals that there is a residual population that settled down inside the corridor; this is readily seen from Fig. 11 that also gives a magnified view of the passage. Since this residual population is still persistent in the passage after a considerable time $(t=9000$ in Fig. 11), we assume that it corresponds to a stationary solution of Eqs. (5-6).

A question arises here as to what can be a response of this residual population to a change in the demographic parameters. Such a change can be a result of the alien species adaptation to their new environment, or it can be a result of some exogenous process such as the global climate change. In either case, the question is whether the population will remain confined inside the passage or it goes extinct, or it may start spreading.

Looking for an answer to the above question, we performed simulations using the residual population as the initial condition for another simulation run with a different value $\beta_{1}$ for the Allee threshold density. We obtain that for $\beta_{1}$ just slightly larger than

\begin{tabular}{|c|c|c|c|}
\hline Boundary conditions in the passage & IC-1 & IC-2 & IC-3 \\
\hline \hline zero-flux (Neumann) & 2340 & 460 & 5090 \\
\hline zero-density (Dirichlet) & 4450 & 3970 & 1240 \\
\hline
\end{tabular}

Table 1: approximate spill-over time required for the population to reach the corridor exit to Habitat 2 for different initial conditions (IC) and different conditions at the passage boundary (cf. Fig. 7). 
$\beta$, e.g. $0.2<\beta_{1} \leq 0.23$, the population remains confined inside the passage and at any moment of time the spatial population distribution of the prey is similar to the one shown in Fig. 11. In case $\beta_{1}$ is sufficiently larger than $\beta$, e.g. $0.24 \leq \beta_{1} \leq 0.3$, the residual population goes extinct. However, in case $\beta_{1}$ is sufficiently smaller than $\beta$, e..g. $0.1 \leq \beta_{1} \leq 0.16$ the prey can start spreading away from the passage into both habitats 1 and 2. As just one example, Fig. 12 show the results obtained in the case where $\beta_{1}=0.1$. Note that the spread takes place following a different scenario compared to the original one; instead of the propagating ring (cf. top-left panel in Fig. 10), it now
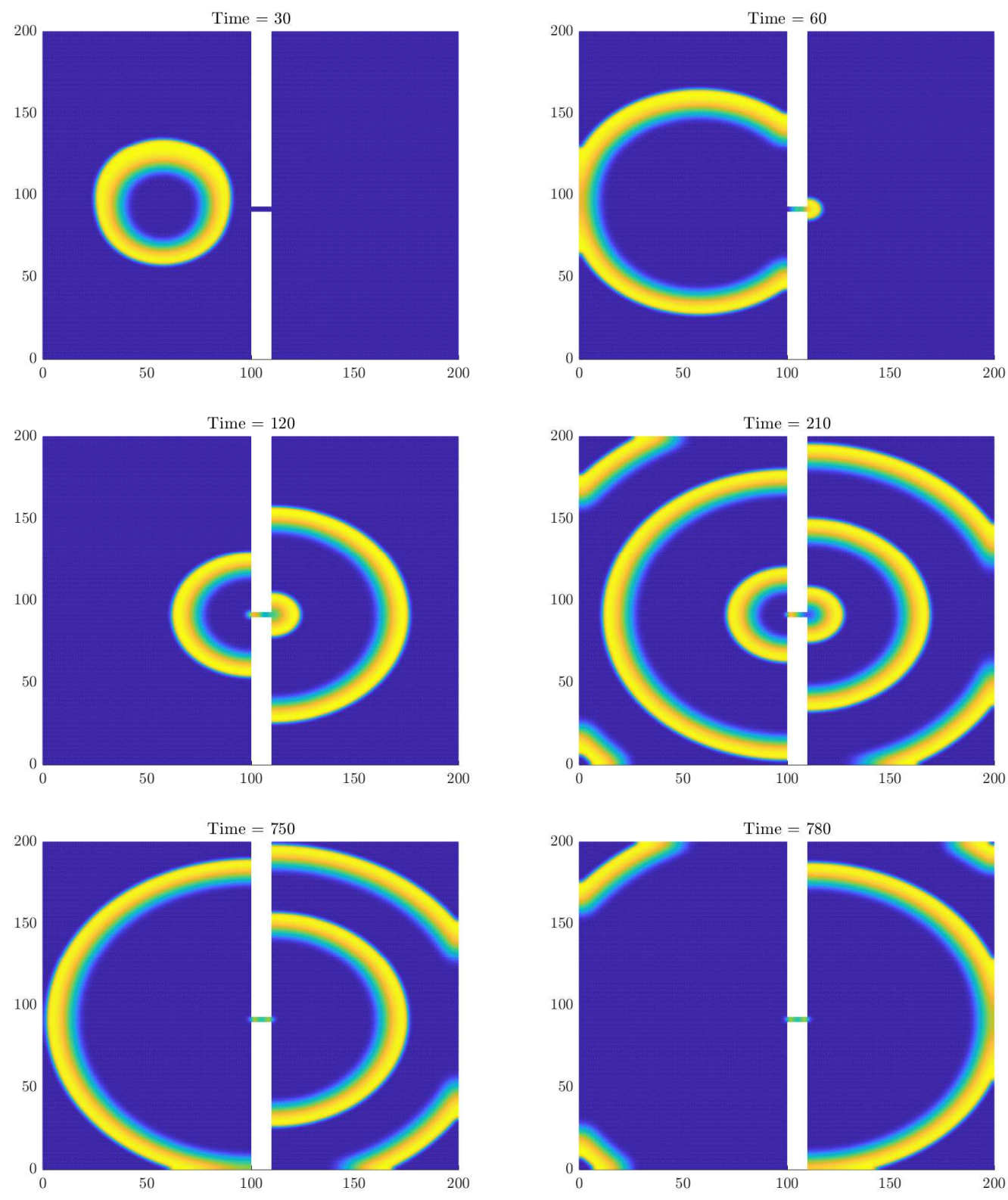

Figure 10: Dynamics of the system resulting in the formation of a stable steady state distribution of the prey species inside the passage that survives after the invading population goes extinct in the rest of the domain. Parameters and the initial conditions are given in the text. 


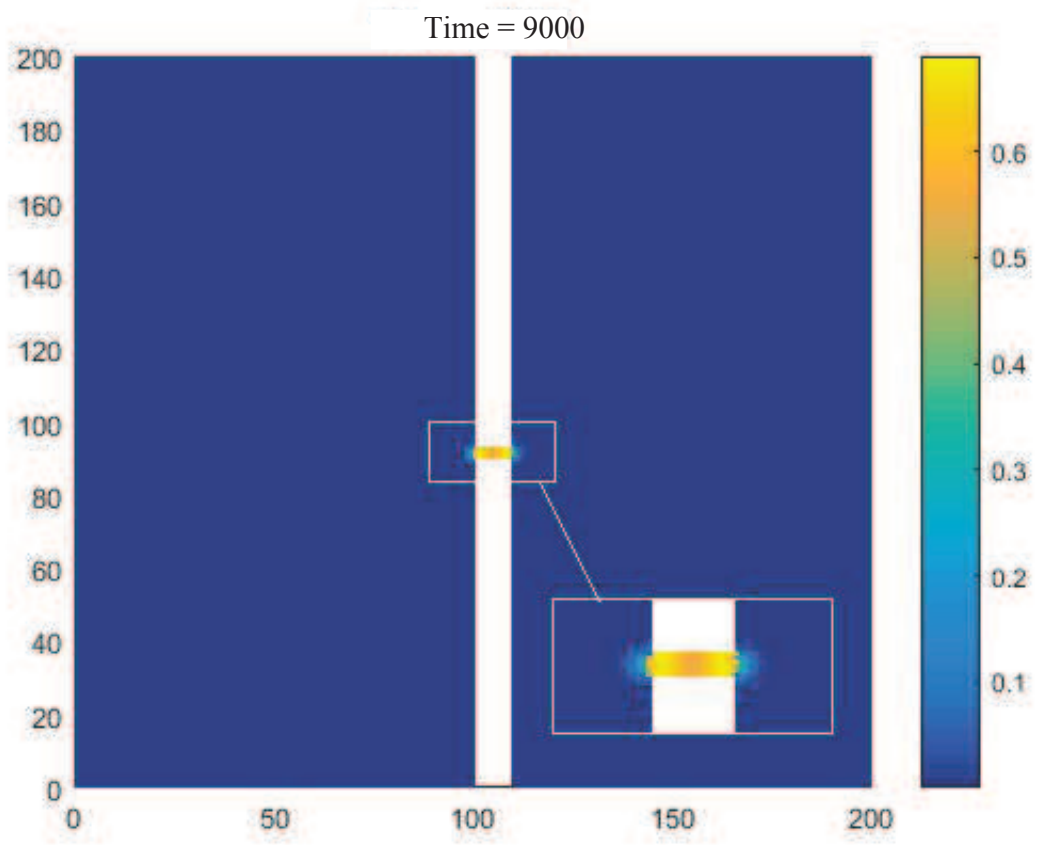

Figure 11: A magnified view of the steady state population distribution inside the passage.
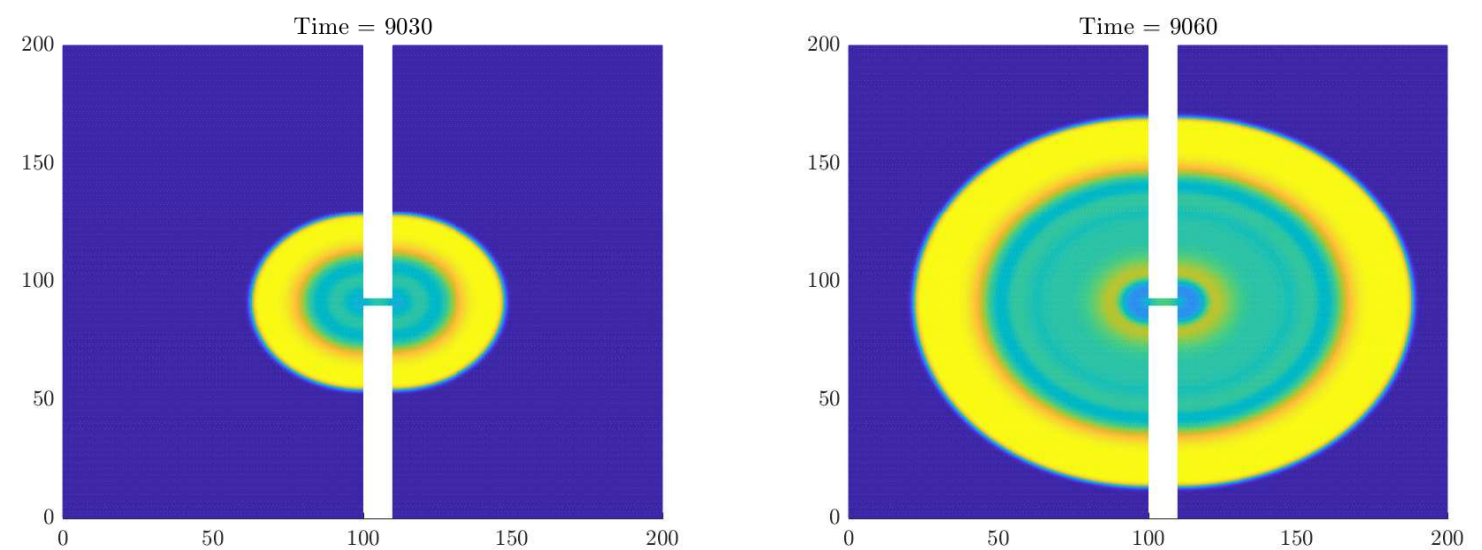

Figure 12: Snapshots of the prey population density at time $t=9030$ (left) and $t=9060$ (right) after the value of the Allee threshold density was changed from the original value $\beta=0.2$ to the new value $\beta=0.1$ at the moment $t=9000$ (see Fig. 11). The residual population that was confined in the passage for a long time not starts spreading into the space.

spreads through a propagating circular front with pattern formation in the wake. This is not surprising as $\beta$ is, along with the predator mortality, a parameter that controls the pattern of invasive spread [27, 35]. 


\section{Discussion and conclusions}

Factors affecting the patterns and rates of alien species spread have been a major focus of research for several decades. A vast majority of studies were concerned with the species proliferation into an "open space" - a relatively uniform environment where all areas, although not necessarily being equally favorable, are accessible to the invading population. However, a realistic landscape can often be strongly heterogeneous combining areas accessible for the invader with inaccessible ones. The effect of the landscape geometry (as given by the size and shape of the inaccessible areas) remains poorly understood. In this paper, we addressed this problem by considering a mathematical model where the population dynamics of an alien species takes place in an H-shaped domain consisting of two large uniform habitats connected by a narrow passage or corridor. For the initial conditions, we considered that the alien species is introduced in a (small) area inside one of the habitats. Our goal was to reveal the effect of the corridor on the species spread, in particular whether the spread from the one habitat to the other could be slowed down or blocked. In terms of the real-world landscape, such a system could account for an alien species spread through a narrow valley or an isthmus. To account for interspecific interactions, we considered a generic prey-predator model which is known to exhibit a rather complex, 'realistic' spatiotemporal dynamics and predicts a variety of different invasion scenarios $[21,24]$.

In reply to the questions bullet-pointed in the introduction, we obtained the following answers:

- The corridor does slow down the spread. It takes the spreading population an extra time to penetrate through the passage. A sufficiently narrow corridor, i.e. its width being less than a certain critical value, blocks the spread so that the invasive population never spills over to the other habitat. Analytical estimates for the critical width are obtained, see Eqs. (14) and (17), that are in good agreement with numerical results;

- The corridor can modify the pattern of spread. An example of this is shown in Fig. 3 where the irregular spatiotemporal pattern emerging behind the propagating front in Habitat 1 changes to a regular (symmetric) spatial population distribution behind the front in Habitat 2. Interestingly, this is exactly opposite to the results of some earlier work [52] where the existence of inaccessible areas - but with a different geometry - changed a regular spatiotemporal pattern to chaos. The regularizing effect of the corridor in our study can be readily explained: having propagated through the narrow passage, the population distribution at the exit to Habitat 2 become symmetric and effectively works as a symmetric initial condition for the population spread in Habitat 2;

- The delay of the spread caused by the corridor is significantly different depending on the pattern of spread. In particular, we obtained that it can be about two orders 
of magnitude longer in case of the patchy spread compared to the spread by the propagating continuous front.

Our results seem to send a potentially important message to the invasion management specialists. Since the corridor tends to impede the invasive species spread, it might be regarded as a possible control strategy. Although it apparently implies a significant alteration of the environment (e.g. to make a natural corridor more narrow), which is likely to be very expensive, we mention here that this strategy is not unrealistic and some examples of large scale environment intervention with the goal to slow down the spread are well known [45]. Importantly, however, based on our results (especially see the last item from the above list) this cannot be a universal approach as its efficiency depends on the pattern of spread: whilst it slows down the invasion considerably in the case of patchy spread, the delay might be rather insignificant in the case of spread via a continuous front. In its turn, this observation highlights the need for a better understanding of the invasion patterns and their more efficient identification including advance adaptive monitoring [37].

Interestingly, although the corridor tends to impede the invasive species spread, it also can promote the invasion if considered on a longer time scale. Once the time is long enough to include an environmental change or species adaptation (e.g. through phenotypic evolution), the corridor can become a source of a secondary invasion (see Section 3.3) hence bringing the alien species back even it may have already disappeared from the rest of the domain. If considered in the context of invasion management, this observation restricts the potential use of the corridor as a factor controlling invasion to a small-tointermediate timescale. From the point of alien species monitoring, it also means that a special attention should be paid to landscape features that can act as corridors, because they may become a refuge for the alien pest.

\section{Acknowledgements}

Dr Andrew Morozov, a member of the Editorial Board, acted as the Chief Editor handling the review processes for this manuscript. The authors are thankful to Prof. Horst Malchow, Prof. Yuri Tyutyunov and Dr Ivo Siekmann for many helpful remarks made on the original version of this work. 


\section{References}

[1] Alharbi WG, Petrovskii SV, 2016. The impact of fragmented habitats size and shape on populations with Allee effect. Mathematical Modelling of Natural Phenomena 11(4), 5-15.

[2] Aronson DG, Weinberger HF, 1978. Multidimensional nonlinear diffusion arising in population genetics. Adv. Math. 30, 33-76.

[3] Andow DA, Kareiva PM, Levin SA, Okubo A, 1990. Spread of invading organisms. Landsc. Ecol. 4, 177-188.

[4] Bell SS, White A, Sherratt JA, Boots M, 2009. Invading with biological weapons: the role of shared disease in ecological invasion. Theor. Ecol. 2, 53-66.

[5] Courchamp F, Clutton-Brock T, Grenfell, B, 1999. Inverse density dependence and the Allee effect. Trends Ecol. Evol. 14, 405-410.

[6] Courchamp F, Berek L, Gascoigne J, 2008. Allee Effects in Ecology and Conservation. Oxford University Press, Oxford.

[7] Davis MB, Calcote RR, Sugita S, Takahara H, 1998. Patchy invasion and the origin of a Hemlock-Hardwoods forest mosaic. Ecology 79, 2641-2659.

[8] Drake JA, Mooney HA, di Castri F, Groves RH, Kruger FJ, 1989. Biological Invasions: A Global Perspective. Wiley, Chichester.

[9] Farashi A, Najafabadi MS, 2015. Modeling the spread of invasive nutrias (Myocastor coypus) over Iran. Ecol. Complex. 22, 59-64.

[10] Fisher RA, 1937. The wave of advance of advantageous genes. Ann. Eugen. 7, 355369.

[11] Jankovic M, Petrovskii SV, 2013 Gypsy moth invasion in North America: a simulation study of the spatial pattern and the rate of spread. Ecol. Compl. 14, 132-144.

[12] Hengeveld R, 1989. Dynamics of Biological Invasions. Chapman and Hall, London.

[13] Kareiva PM, 1990. Population dynamics in spatially complex environments: theory and data. Philos Trans R Soc Lond B 330, 175-190.

[14] Keitt TH, Lewis MA, Holt RD, 2001. Allee effects, invasion pinning, and species' borders. Am. Nat. 157, 203-216.

[15] Keller RP, Lodge DM, Lewis MA, Shogren JF, 2009. Bioeconomics of Invasive Species: Integrating Ecology, Economics, Policy, and Management. Oxford University Press, Oxford. 
[16] Kenis M, Vaamonde CL, 1998. Classical biological control of the gypsy moth, Lymantria dispar (L.), in: North America: prospects and new strategies. Population Dynamics, Impacts, and Integrated Management of Forest Defoliating Insects, pp. 213-221. Eds. McManus ML, Liebhold AM. USDA Forest Service General Technical Report NE-247.

[17] Kot M, Lewis MA, van den Driessche P, 1996. Dispersal data and the spread of invading organisms. Ecology 77, 2027-2042.

[18] Kolmogorov AN, Petrovskii IG, Piskunov NS, 1937. A study of the diffusion equation with increase in the quantity of matter, and its application to a biological problem. Bull. Moscow Univ. Math. Ser. A 1, 1-25.

[19] Lewis MA, 2000. Spread rate for a nonlinear stochastic invasion. J. Math. Biol. 41, 430-454.

[20] Lewis MA, Kareiva P, 1993. Allee dynamics and the spread of invading organisms. Theor. Popul. Biol. 43, 141-158.

[21] Lewis MA, Petrovskii SV, Potts J, 2016. The Mathematics Behind Biological Invasions. Interdisciplinary Applied Mathematics, Vol. 44. Springer, Berlin.

[22] Liebhold AM, Halverson JA, Elmes GA, 1992. Gypsy moth invasion in North America: a quantitative analysis. J. Biogeogr. 19, 513-520.

[23] Mack RN, 1981 Invasion in Bromus tectorum L. into western North America: an ecological chronicle. Agro-Ecosystems 7, 145-165.

[24] Malchow H, Petrovskii SV, Venturino E, 2008. Spatiotemporal Patterns in Ecology and Epidemiology: Theory, Models, Simulations. CRC Press, Boca Raton.

[25] Maynard Smith J, 1974. Models in Ecology. Cambridge University Press, Cambridge.

[26] Mistro DC, Rodrigues LAD, Petrovskii SV, 2012. Spatiotemporal complexity of biological invasion in a space- and time-discrete predator-prey system with the strong Allee effect. Ecol. Compl. 9: 16-32.

[27] Morozov AY, Petrovskii SV, Li BL, 2006. Spatiotemporal complexity of patchy invasion in a predator-prey system with the Allee effect. J. Theor. Biol. 238: 18-35.

[28] Murray JD, 1989. Mathematical Biology. Springer, Berlin.

[29] Okubo A, Maini PK, Williamson MH, Murray JD, 1989. On the spatial spread of the grey squirrel in Britain. Proc. R. Soc. B 238, 113-125.

[30] Owen MR, Lewis MA, 2001. How predation can slow, stop or reverse a prey invasion. Bull. Math. Biol. 63, 655-684. 
[31] Pascual M, 2005. Computational ecology: from the complex to the simple and back. PLoS Comp. Biol. 1, e18.

[32] Petrovskii SV, 1998. Modeling of open-sea ecological impact: impact wave localization and pattern formation. Environ. Model. Assess. 3, 127-133.

[33] Petrovskii SV, McKay K, 2010. Biological invasion and biological control: A case study of the gypsy moth spread. Aspects of Applied Biology 104, 37-48.

[34] Petrovskii SV, Petrovskaya NB, 2012. Computational ecology as an emerging science. Interface Focus 2, 241-254.

[35] Petrovskii SV, Morozov AY, Li BL, 2005. Regimes of biological invasion in a predatorprey system with the Allee effect. Bulletin of Mathematical Biology 67, 637-661.

[36] Petrovskii SV, Morozov AY, Venturino E, 2002. Allee effect makes possible patchy invasion in a prey-predator system. Ecol. Lett. 5: 345-352.

[37] Petrovskii SV, Petrovskaya N, Bearup D, 2014. Multiscale approach to pest insect monitoring: Random walks, pattern formation, synchronization, and networks. Physics of Life Reviews 11, 467-525.

[38] Petrovskii SV, Malchow H, Hilker FM, Venturino E, 2005. Patterns of patchy spread in deterministic and stochastic models of biological invasion and biological control. Biological Invasions 7: 771-793.

[39] Pimentel D, Ed., 2002. Biological Invasions: Economic and Environmental Costs of Alien Plant, Animal, and Microbe Species. CRC Press, Boca Raton.

[40] Protter MH, Weinberger HF, 1984. Maximum Principles in Differential Equations. Springer, New York.

[41] Richardson DM, Ed., 2011. Fifty Years of Invasion Ecology: The Legacy of Charles Elton. Wiley, Chichester.

[42] Richtmyer RD, Morton KW, 1967. Difference Methods for Initial Value Problems. Interscience, New York.

[43] Rodrigues LAD, Mistro DC, Cara ER, Petrovskaya NB, S.V.Petrovskii SV, 2015. Patchy invasion of stage-structured alien species with short-distance and longdistance dispersal. Bulletin of Mathematical Biology, 77, 1583-1619.

[44] Sandlund OT, Schei PJ, Viken A, 2001. Invasive Species and Biodiversity Management. Kluwer Academic, Dordrecht.

[45] Sharov AA, Leonard D, Liebhold AM, Roberts EA, Dickerson W, 2002. Slow the spread: a national program to contain the gypsy moth. Journal of Forestry 100, 30-36. 
[46] Sherratt JA, Lewis MA, Fowler AC, 1995. Ecological chaos in the wake of invasion. Proc. Natl. Acad. Sci. USA 92, 2524-2528.

[47] Sherratt JA, Eagan BT, Lewis MA, 1997. Oscillations and chaos behind predatorprey invasion: mathematical artifact or ecological reality? Phil. Trans. R. Soc. Lond. B 352, 21-38.

[48] Shigesada N, Kawasaki K, Teramoto E, 1986. Traveling periodic waves in heterogeneous environments. Theor. Popul. Biol. 30, 143-160.

[49] Shigesada N, Kawasaki K, 1997. Biological Invasions: Theory and Practice. Oxford University Press, Oxford.

[50] Shigesada N, Kawasaki K, Takeda Y, 1995. Modeling stratified diffusion in biological invasions. Am. Nat. 146, 229-251.

[51] Skellam JG, 1951. Random dispersal in theoretical populations. Biometrika 38, 196218.

[52] Smith MJ, Sherratt JA, Armstrong NJ, 2008. The effects of obstacle size on periodic travelling waves in oscillatory reaction-diffusion equations. Proc R Soc A 464, 365390.

[53] Volpert AI, Khudyaev SI, 1985. Analysis in Classes of Discontinuous Functions and Equations of Mathematical Physics. Nijoff, Dordrecht.

[54] Volpert V, Petrovskii SV, 2009. Reaction-diffusion waves in biology. Physics of Life Reviews 6, 267-310.

[55] Wang ME, Kot M, 2001. Speeds of invasion in a model with strong or weak Allee effects. Math. Biosci. 171, 83-97.

[56] Andy White, personal communication. http://www.macs.hw.ac.uk/ awhite/

[57] Williamson M, 1996. Biological Invasions. Chapman \& Hall, London. 